\title{
First molecular detection of Anaplasma phagocytophilum in the hard tick Rhipicephalus haemaphysaloides in Taiwan
}

\author{
Chi-Chien Kuo ${ }^{1,2,3} \cdot$ Jing-Lun Huang ${ }^{2,4} \cdot$ Chia-Hao Chien ${ }^{2} \cdot$ Han-Chun Shih ${ }^{2}$. \\ Hsi-Chieh Wang ${ }^{2}$ (D)
}

Received: 6 June 2018 / Accepted: 13 August 2018 / Published online: 16 August 2018

(c) Springer Nature Switzerland AG 2018

\begin{abstract}
Anaplasma phagocytophilum is transmitted mainly by hard ticks and can cause potentially fatal granulocytic anaplasmosis in humans, but its occurrence in ticks in Taiwan has never been investigated although this pathogen has been detected in Taiwanese rodents before. Ticks collected from small mammals in Hualien, eastern Taiwan, were assayed for Anaplasma infections; infections of Rickettsia and Apicomplexa protozoans were also studied. Of the 270 individually assayed Rhipicephalus haemaphysaloides ticks, A. phagocytophilum was identified in a nymphal tick. Parasites most similar to Anaplasma bovis, Rickettsia rickettsii, Rickettsia sp. TwKM01, and at least seven apicomplexan species (including genera Cryptosporidium, Hepatozoon, and Theileria) were also identified. This study shows that A. phagocytophilum does occur in the hard tick in Taiwan, although whether R. haemaphysaloides can vector this pathogen remains to be determined. This work also reveals a high diversity of tick-borne bacteria and protozoans circulating in a small region and calls for further research on their potential risks for human health.
\end{abstract}

Keywords Anaplasma phagocytophilum - Rickettsia $\cdot$ Apicomplexa · Ticks · Tick-borne diseases · Taiwan

\section{Introduction}

Anaplasma phagocytophilum, the etiological agent of potentially fatal granulocytic anaplasmosis in humans and tick-borne fever in ruminants is transmitted mainly by hard ticks, particularly the Ixodes species (Woldehiwet 2000; Stuen et al. 2013). This pathogen can

Hsi-Chieh Wang

sjwang1019@gmail.com

1 Department of Life Science, National Taiwan Normal University, Taipei, Taiwan

2 Center for Diagnostics and Vaccine Development, Centers for Disease Control, Ministry of Health and Welfare, Taipei, Taiwan

3 Department of Wildlife, Fish, and Conservation Biology, University of California, One Shields Avenue, Davis, CA 95616, USA

4 Biological Sciences, Institute for Life Sciences, University of Southampton, Southampton, UK 
be transmitted transstadially but not transovarially in ticks; vertebrate reservoir hosts are necessary for infecting larval ticks to maintain A. phagocytophilum circulation in natural settings (Stuen et al. 2013).

Anaplasma phagocytophilum is widespread around the globe and is emerging in several places, including East Asia (Stuen et al. 2013). Human cases of granulocytic anaplasmosis have been confirmed with molecular methods in China (Zhang et al. 2008), Japan (Ohashi et al. 2013), and Korea (Kim et al. 2014), but not in Taiwan. However, that fact that $A$. phagocytophilum has been detected from rodents in Taiwan (Masuzawa et al. 2014) suggests that this pathogen also circulates in Taiwan and local people are at risks of getting infected with the disease. Previous study on Anaplasma infection in Taiwan has identified A. platys in hard ticks (Rhipicephalus sanguineus) collected from dogs (Yuasa et al. 2017), but detection of $A$. phagocytophilum has never been attempted in ticks collected from small mammals. This is despite the fact that $A$. phagocytophilum was found on Taiwanese rodents (Masuzawa et al. 2014) and ticks can vector this pathogen among rodent hosts and likely also to humans. One purpose of this study was thus to investigate A. phagocytophilum infection in ticks collected from small mammals. In addition, although infection of Rickettsia spp., the causative agent of human spotted fever, is widespread in ticks collected from shrews and rodents in Taiwan (Kuo et al. 2015a), the study focuses on geographical variation across Taiwan (Kuo et al. 2015a), leaving specific regions of Taiwan less intensively studied. One study that focuses on Rickettsia infection in ticks in eastern Taiwan (Hualien) reported only prevalence of infection but not the identity of the Rickettsia species (Kuo et al. 2012). Likewise, protozoans in hard ticks have rarely been investigated intensively in Taiwan although some disease-causing species, such as Babesia microti that causes babesiosis in humans, are also detected in rodents in Taiwan (Saito-Ito et al. 2008; Chao et al. 2017). Therefore, the other aim of this study is to intensively investigate Rickettsia and protozoan infection in ticks in Hualien, where ticks are common on shrews and rodents (Kuo et al. 2011a).

\section{Materials and methods}

\section{Study area and tick collection}

Ticks were collected during a surveillance project of ectoparasites on small mammals in Taiwan (Kuo et al. 2011a, b, 2012). Briefly, small mammal traps were set up in Ji-an, Shou-feng and Fong-lin townships in Hualien County, eastern Taiwan, during 2007 and 2008. Ticks were removed from trapped shrews and rodents, preserved in $70 \%$ ethanol, and stored at $-70{ }^{\circ} \mathrm{C}$ for later pathogen assay. Species of ticks were identified following published keys (Teng and Jiang 1991) and confirmed by comparing 12S rDNA sequences with known species (Beati and Keirans 2001).

\section{Pathogen detection in ticks}

Each tick was placed in a $2 \mathrm{ml}$ eppendorf tube and crushed by shaking with steel grinding balls in a bead beater (TissueLyser II, Qiagen) for $2.5 \mathrm{~min}$ at a frequency of 30 oscillations per second, then individually assayed for infection of bacteria (Anaplasma, Rickettsia), and protozoans (Apicomplexa). Primers and methods for amplifying fragments of these organisms were provided in Table 1. Positive samples were sequenced twice in each direction 


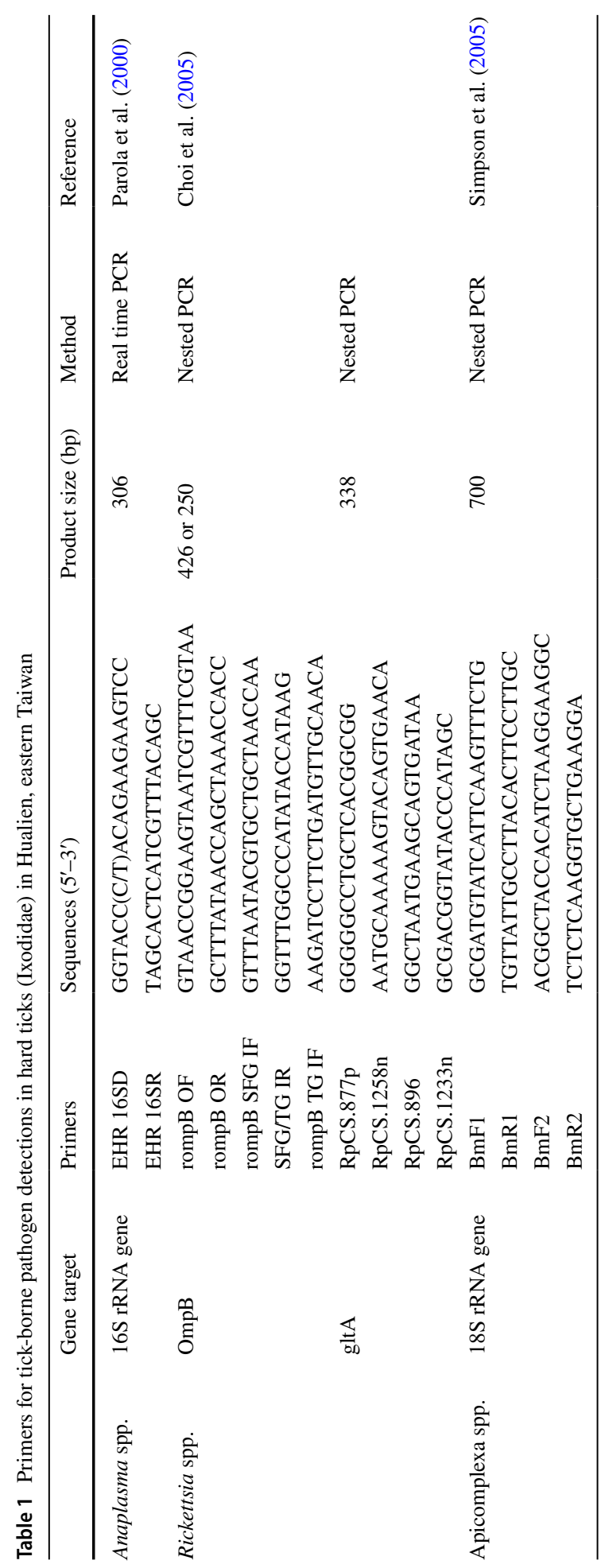


and potential parasitic species were identified by resemblance to known species using the Basic Local Alignment Search Tool.

\section{Results}

\section{Tick collection}

A total of 270 Rhipicephalus haemaphysaloides ticks were assayed individually, including 155 larvae and 115 nymphs (no adult $R$. haemaphysaloides were collected). These ticks were collected from eight small mammal species, including two shrew (Crocidura attenuate, Suncus murinus) and six rodent species (Apodemus agrarius, Bandicota indica, Mus caroli, M. musculus, Rattus exulans, R. losea) (Table 2).

\section{Pathogen assay}

Two Anaplasma spp. were identified, including one A. phagocytophilum (100\% identity to A. phagocytophilum, GenBank accession number: MG519284.1) in a nymphal R. haemaphysaloides collected from A. agrarius (prevalence 0.4\%) in Shou-feng and 19 detections of bacteria most similar to Anaplasma bovis in 6 larvae and 13 nymphs (prevalence $7.0 \%$ ) collected from A. agrarius and $R$. losea (Table 2). Likewise, two Rickettsia spp. were recognized, including 16 detections of $R$. rickettsia-like bacteria in 7 larvae and 9 nymphs (prevalence 5.9\%) from A. agrarius, B. indica, and R. losea, and 74 detections of Rickettsia sp. TwKM01-like bacteria in 37 larvae and 37 nymphs (prevalence 27.4\%) collected from all rodent species except for M. musculus (Table 2). Lastly, at least seven Apicomplexa species were identified, including nucleotide sequences most similar to two Cryptosporidium spp. (C. galli, C. struthionis), four Hepatozoon spp. (Hepatozoon sp. DB2231, Hepatozoon sp. HepBiCM001, Hepatozoon sp. pty01 po, Hepatozoon sp. YLW-2014 isolate), and two detections of Theileria genus with unknown species. These Apicomplexa species were mostly identified once (prevalence 0.4\%), except for Hepatozoon sp. DB2231 that were detected twice (prevalence 0.7\%) and Hepatozoon sp. YLW-2014 isolate that were detected five times (prevalence 1.9\%). These 13 Apicomplexa detections occurred to two larvae and 11 nymphs removed from A. agrarius, $M$. caroli, and $R$. losea (Table 2).

\section{Discussion}

In this study, A. phagocytophilum was identified in a hard tick for the first time in Taiwan. In addition, another Anaplasma sp. (A. bovis) also circulates locally. Lastly, we detected a moderate prevalence of Rickettsia infection and several Apicomplexa protozoan species. These results demonstrate a variety of tick-borne bacteria and protozoans circulating in eastern Taiwan and could pose health risks to local people.

We detected A. phagocytophilum in a $R$. haemaphysaloides tick collected from A. agrarius rodent, showing that $A$. phagocytophilum not only occurs in central Taiwan and Kinmen Island (Masuzawa et al. 2014) but also in eastern Taiwan. Furthermore, the pathogen was detected in $R$. haemaphysaloides that also bites humans (Guglielmone et al. 2014). It should be noted, nevertheless, that the detection of A. phagocytophilum doesn't mean that the tick can certainly vector the pathogen because the assayed tick was retrieved from the 


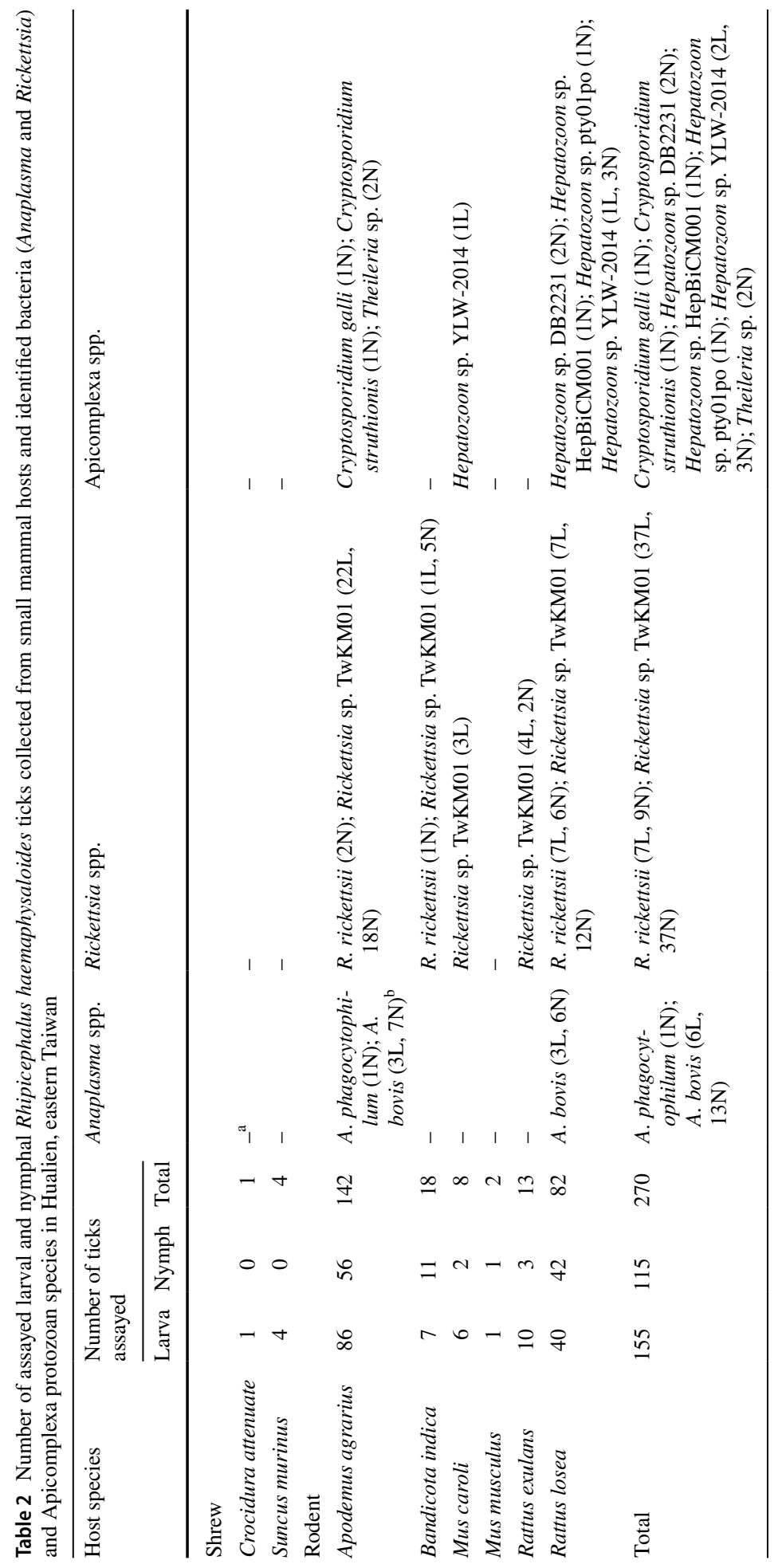

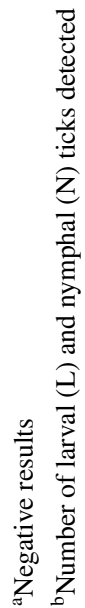


rodent host and A. phagocytophilum might come from un-digested blood meal, particularly when $A$. agrarius rodent is implicated as a main reservoir of A. phagocytophilum in other countries, such as China (Cao et al. 2006) and Korea (Kim et al. 2006). However, because A. phagocytophilum is transmitted mainly by ticks (Stuen et al. 2013) and the local tick fauna is dominated by R. haemaphysaloides (>99\%, Kuo et al. 2011a), R. haemaphysaloides is the most likely candidate vector for propagating A. phagocytophilum transmission in our study area.

Likewise, A. bovis has been detected in small mammals in central Taiwan and Kinmen Island before (Masuzawa et al. 2014) and similar bacteria were detected in the hard ticks for the first time in Taiwan. This study and Masuzawa et al. (2014) both revealed the existence and only the existence of $A$. phagocytophilum and A. bovis in small mammals and associated ticks in Taiwan; besides, the latter bacteria was more common than former one. A. bovis has been identified in ruminants and several other mammals groups around the world (Ben Said et al. 2015), including China (Liu et al. 2012), Japan (Yoshimoto et al. 2010), and Korea (Doan et al. 2013), so it might not be surprising that this pathogen also occurs in Taiwan. Likewise, the 19 detections of $A$. bovis came from $R$. haemaphysaloides ticks, which might be the local vector of $A$. bovis.

Rickettsia spp. commonly occur in small mammals (Kuo et al. 2015b) and associated ticks (Kuo et al. 2015a) across Taiwan. In this study, we found that Rickettsia sp. TwKM01like bacteria was common in R. haemaphysaloides ticks in Hualien (prevalence 27.4\%). Previous studies on Rickettsia infection in the same region have detected Rickettsia sp. TwKM01 in small mammals (Kuo et al. 2015b) but not in ticks (Kuo et al. 2015a), likely due to the small number of ticks $(\mathrm{n}=10)$ being assayed in Kuo et al. (2015a). Rickettsia sp. TwKM01 was first recognized in $R$. haemaphysaloides in Hualien and Kinmen Island and its pathogenicity remains to be determined (Tsui et al. 2007), but together these studies show that Rickettsia sp. TwKM01 was identified mostly in $R$. haemaphysaloides although it has also been detected in Ixodes granulatus ticks, one time each in Kinmen Island and Penghu Island (Kuo et al. 2015a).

Babesia microti that can cause babesiosis in humans was not found in this study; instead, we identified $\geq 7$ protozoan species, including those in Cryptosporidium, Hepatozoon, and Theileria. Worldwide, these three genera include some species of medical and veterinary importance, such as Cryptosporidium hominis in humans, Hepatozoon canis in canids, and Theileria annulata in the livestock (Bouzid et al. 2013; Sumrandee et al. 2015). Pathogenicity of the $\geq 7$ apicomplexan species recognized herein is unknown, but the many species identified in a small region calls for a more intensive and extensive study on this neglected group of parasites in Taiwan.

Acknowledgements We are indebted to those who helped with the field work. This study was financially supported by Taiwan Centers for Disease Control (DOH96-DC-2019) and University of California, Davis. All animal handling procedures were approved by the University of California Davis Animal Use and Care Administrative Advisory Committee and fulfilled Taiwanese legislative requirements.

\section{References}

Beati L, Keirans JE (2001) Analysis of the systematic relationships among ticks of the genera Rhipicephalus and Boophilus (Acari: Ixodidae) based on mitochondrial 12S ribosomal DNA gene sequences and morphological characters. J Parasitol 87:32-48

Ben Said M, Belkahia H, Karaoud M, Bousrih M, Yahiaoui M, Daaloul-Jedidi M, Messadi L (2015) First molecular survey of Anaplasma bovis in small ruminants from Tunisia. Vet Microbiol 179:322-326 
Bouzid M, Hunter PR, Chalmers RM, Tyler KM (2013) Cryptosporidium pathogenicity and virulence. Clin Microbiol Rev 26:115-134

Cao WC, Zhan L, He J, Foley JE, Sj DEV, Wu XM et al (2006) Natural Anaplasma phagocytophilum infection of ticks and rodents from a forest area of Jilin Province, China. Am J Trop Med Hyg 75:664-668

Chao LL, Yu WC, Shih CM (2017) First detection and molecular identification of Babesia microti in Rattus losea captured from the offshore Kinmen Island of Taiwan. Ticks Tick Borne Dis 8:313-319

Choi YJ, Jang WJ, Kim JH, Ryu JS, Lee SH, Yark KH et al (2005) Spotted fever group and typhus group rickettsioses in humans, South Korea. Emerg Infect Dis 11:237-244

Doan HT, Noh JH, Choe SE, Yoo MS, Kim YH, Reddy KE et al (2013) Molecular detection and phylogenetic analysis of Anaplasma bovis from Haemaphysalis longicornis feeding on grazing cattle in Korea. Vet Parasitol 196:478-481

Guglielmone AA, Robbins RG, Apanaskevich DA, Petney TN, Estrada-Pena A, Horak IG (2014) The hard ticks of the world. Springer, Dordrecht

Kim CM, Yi YH, Yu DH, Lee MJ, Cho MR, Desai AR et al (2006) Tick-borne rickettsial pathogens in ticks and small mammals in Korea. Appl Environ Microbiol 72:5766-5776

Kim KH, Yi J, Oh WS, Kim NH, Choi SJ, Choe PG et al (2014) Human granulocytic anaplasmosis, South Korea, 2013. Emerg Infect Dis 20:1708-1711

Kuo CC, Huang CL, Wang HC (2011a) Identification of potential hosts and vectors of scrub typhus and tick-borne spotted fever group rickettsiae in eastern Taiwan. Med Vet Entomol 25:169-177

Kuo CC, Wang HC, Huang CL (2011b) The potential effect of exotic pacific rats Rattus exulans on vectors of scrub typhus. J Appl Ecol 48:192-198

Kuo CC, Huang JL, Shu PY, Lee PL, Kelt DA, Wang HC (2012) Cascading effect of economic globalization on human risks of scrub typhus and tick-borne rickettsial diseases. Ecol Appl 22:1803-1816

Kuo CC, Shu PY, Mu JJ, Lee PL, Wu YW, Chung CK, Wang HC (2015a) Widespread Rickettsia spp. infections in ticks in Taiwan. J Med Entomol 52:1096-1102

Kuo CC, Shu PY, Mu JJ, Wang HC (2015b) High prevalence of Rickettsia spp. infections in small mammals in Taiwan. Vector Borne Zoonotic Dis 15:13-20

Liu Z, Ma M, Wang Z, Wang J, Peng Y, Li Y et al (2012) Molecular survey and genetic identification of Anaplasma species in goats from central and southern China. Appl Environ Microbiol 78:464-470

Masuzawa T, Uchishima Y, Fukui T, Okamoto Y, Pan MJ, Kadosaka T et al (2014) Detection of Anaplasma phagocytophilum and Anaplasma bovis in small wild mammals from Taichung and Kinmen Island, Taiwan. Jpn J Infect Dis 67:111-114

Ohashi N, Gaowa Wuritu, Kawamori WuD, Yoshikawa Y et al (2013) Human granulocytic anaplasmosis, Japan. Emerg Infect Dis 19:289-292

Parola P, Roux V, Camicas JL, Baradji I, Brouqui P, Raoult D (2000) Detection of ehrlichiae in African ticks by polymerase chain reaction. Trans R Soc Trop Med Hyg 94:707-708

Saito-Ito A, Takada N, Ishiguro F, Fujita H, Yano Y, Ma XH et al (2008) Detection of Kobe-type Babesia microti associated with Japanese human babesiosis in field rodents in central Taiwan and southeastern mainland China. Parasitology 135:691-699

Simpson VR, Panciera RJ, Hargreaves J, McGrarry JW, Scholes SFE, Bown KJ, Birtles BR (2005) Myocarditis and myositis due to infection with Hepatozoon species in pine martens (Martes martes) in Scotland. Vet Rec 156:442-446

Stuen S, Granquist E, Silaghi C (2013) Anaplasma phagocytophilum-a widespread multi-host pathogen with highly adaptive strategies. Front Cell Infect Microbiol 3:31

Sumrandee C, Baimai V, Trinachartvanit W, Ahantarig A (2015) Hepatozoon and Theileria species detected in ticks collected from mammals and snakes in Thailand. Ticks Tick Borne Dis 6:309-315

Teng KF, Jiang ZJ (1991) Economic Insect Fauna of China. Fasc 39. Acari: Ixodidae. Editorial Committee of Fauna Sinica, Academic Sinica, Science Press, Beijing

Tsui PY, Tsai KH, Weng MH, Hung YW et al (2007) Molecular detection and characterization of spotted fever group rickettsiae in Taiwan. Am J Trop Med Hyg 77:883-890

Woldehiwet Z (2000) The natural history of Anaplasma phagocytophilum. Vet Parasitol 167:108-122

Yoshimoto K, Matsuyama Y, Matsuda H, Sakamoto L, Matsumoto K, Yokoyama N et al (2010) Detection of Anaplasma bovis and Anaplasma phagocytophilum DNA from Haemaphysalis megaspinosa in Hokkaido, Japan. Vet Parasitol 168:170-172

Yuasa Y, Tsai YL, Chang CC, Hsu TH, Chou CC (2017) The prevalence of Anaplasma platys and a potential novel Anaplasma species exceed that of Ehrlichia canis in asymptomatic dogs and Rhipicephalus sanguineus in Taiwan. J Vet Med Sci 79:1494-1502

Zhang L, Liu Y, Ni D, Li Q, Yu Y, Yu XJ et al (2008) Nosocomial transmission of human granulocytic anaplasmosis in China. JAMA 300:2263-2270 